\title{
Feasibility and barriers of treating clubfeet in four countries
}

\author{
Suzanne F. M. van Wijck ${ }^{1}$ A. Marjanne Oomen ${ }^{2}$ Huub J. L. van der Heide ${ }^{1}$
}

Received: 21 March 2015 / Accepted: 29 March 2015 /Published online: 5 May 2015

(C) The Author(s) 2015. This article is published with open access at Springerlink.com

\begin{abstract}
Purpose The purpose of this study was to elucidate why neglected clubfeet still exists despite the availability of a highly (cost)-effective treatment- the Ponseti treatment.

Methods A qualitative and partly quantitative study with semi-structured interviews was conducted in four countries: the Netherlands, South Africa, Argentina and Indonesia with both caregivers, mostly parents, of children with clubfoot and practitioners treating clubfoot. The topics discussed with the caregivers $(n=51)$ were the conceptions of the cause of clubfeet, received information, accessibility, financial aspects, and social stigma. With the practitioners $(n=11)$ the focus of the interviews was the treatment protocol and finance.

Results Several barriers towards the start of the treatment were highlighted. At all places treatment was financed by the government, insurance or charity. Nevertheless, the cost of transport and missed working days formed a barrier, although there is a large difference between and within countries. Poverty, long travel duration, and beliefs of supernatural causes for the clubfoot result in delay in the start of treatment. Conclusion These are problems we need to address when making effective treatment available for every child to
\end{abstract}

Huub J. L. van der Heide

H.J.L.van_der_Heide@lumc.nl

Suzanne F. M. van Wijck

suzannevanwijck@hotmail.com

A. Marjanne Oomen

marjanneoomen@icloud.com

1 Leiden University Medical Centre (LUMC), Albinusdreef 2, 2333

ZA Leiden, The Netherlands

2 Yayasan Stepping Stones Bali, Jalan Pura Wingin, Kalibukbuk, Singaraja, Indonesia diminish the burden of neglected clubfoot; especially accessible clinics in rural areas can be a good alternative to highly specialized hospitals in large cities. We as a community should try to find and overcome the barriers to treat these patients, because we have a relatively easy and highly costeffective treatment option which can be given by trained nonphysicians supervised by an interested medical doctor.

Keywords Clubfoot · Qualitative research · International · Ponseti $\cdot$ Low-income countries

\section{Introduction}

The idiopathic clubfoot is the most common congenital musculoskeletal deformity and affects about one in 1,000 newborns. The Ponseti treatment provides, in over $95 \%$ of the cases, a foot which is completely functional and pain free when started before the walking age; however, most of the studies with this high amount of successful treatment are from industrialized countries [1, 2]. In the English literature there are numerous examples present of successful introduction of the Ponseti method in the low- and middle-income setting [3-6]. Given the relatively simple and highly cost-effective treatment, the question arises as to why neglected clubfoot still exists.

Ignacio Ponseti stated that the ideal start of the Ponseti treatment is within the first few weeks after birth, with exception of premature born babies. When treatment is delayed, it becomes progressively more difficult to correct the deformity [7]. The likelihood of the requirement of extensive foot surgery dramatically increases, with less effectiveness, more complications and higher costs (Figs. 1 and 2) [3]. When not treated at all, the neglected clubfoot causes the child to grow up with a disability that has problems with societal stigma, is 
Fig. 1 A 13-year-old male high school student with bilateral neglected clubfeet. a AP view. $\mathbf{b}$ PA view
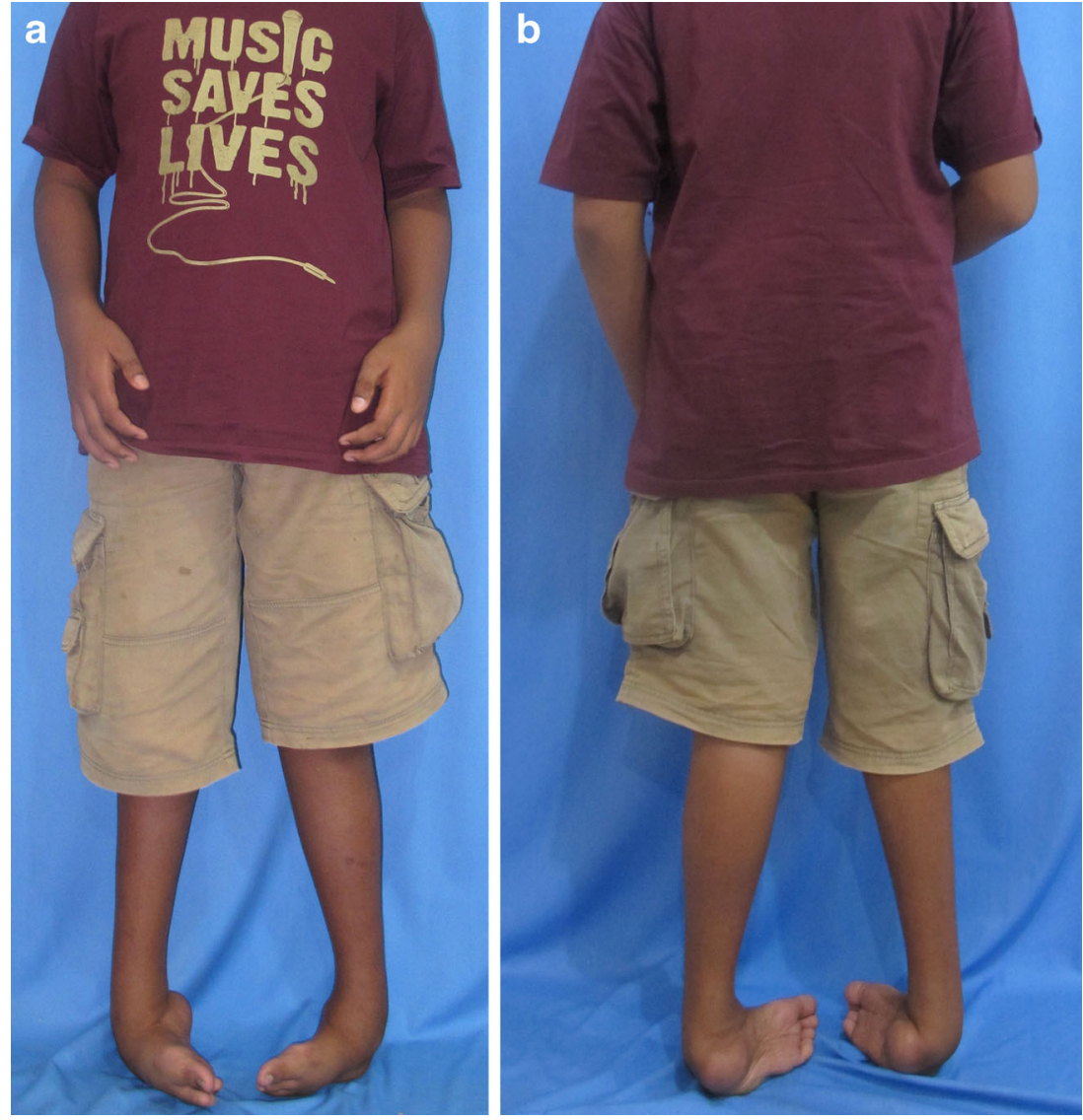

severely painful, reduces mobility, and threatens their potential productivity [8].

The purpose of the present study was to elucidate the reason why neglected clubfeet, considered the most severe of the congenital orthopaedic disorders, still exists despite the availability of a highly effective and cost efficient treatment. The goal of the research was to identify when the treatment starts and why at that time in order to understand more about contextual influences in different nations around the globe.

\section{Research methods}

Qualitative methodology was used for data collection in public hospitals in the capital cities of the Netherlands, South
Africa and Argentina and two rehabilitation clinics on Bali and one on Sumatra, both Indonesian islands. Structures for interviews were composed of a mix of closed and open-ended questions. Two sorts of interviews were used, one for caregivers, who was the mother in most cases, of patients with clubfoot and another for the healthcare professionals who provide treatment for clubfoot. All the interviews were conducted face-to-face by two of the authors (SvW and AO). The topics discussed in the interviews with the parents were: age at the start of treatment, walking ability of the children, family situation, barriers to seek or continue treatment, travel and transport, financial situation, investments in the treatment, diagnosis and information received about the clubfoot, social stigma and beliefs about the cause of clubfoot. In researching the beliefs we distinguished between belief in a biological/
Fig. 2 An eight-year-old boy with neglected clubfeet. a Non weightbearing AP. b

Weightbearing AP
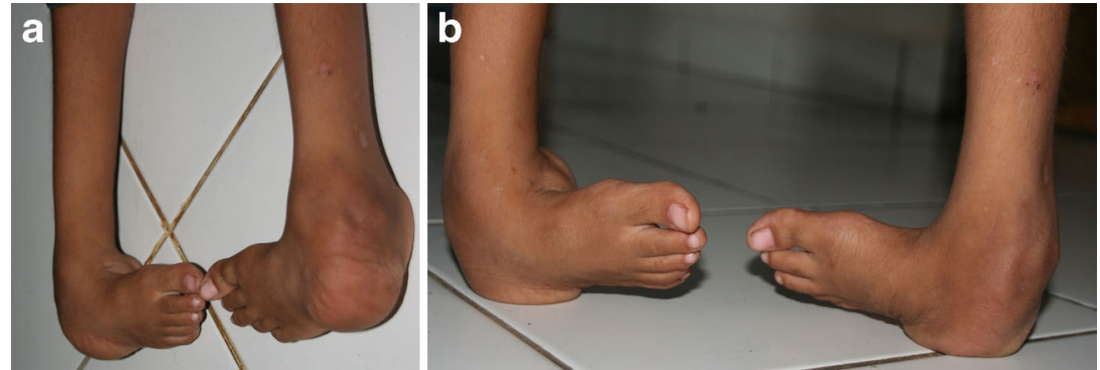
biomechanical reason that the child has a clubfoot, or supernatural beliefs, by which is meant that the clubfoot is caused by evil spirits, a higher spiritual force like, for example, God, Allah, Jaweh, etc. or that the disability is a result of misdeeds of the family. With the healthcare professionals the topics of the interviews were: treatment protocol, finance, responsibility, bracing protocol and the occurrence of neglected clubfoot.

Consecutive patients and/or their caregivers being treated for a clubfoot were asked to participate in this study during their visits to the outpatient clinic; none of the caregivers or children who were asked to participate in the study refused.

To make comparison possible between the countries, as previously done by Cavanagh et al. [9], local currency costs were converted to international dollars, making use of the 2011 and 2012 estimates of the Purchasing Power Parity (PPP) conversion factor published by the World Bank. Purchasing power parity conversion factor is the number of units of a country's currency required to buy the same amounts of goods and services in the domestic market as one U.S. dollar would buy in the United States. For the Netherlands this is 0.83, for South Africa 4.95, for Argentina 2.66 and for Indonesia 3705.04 [10]. To convert costs or the income of the family in international dollars, the following formula was used:

Amount in local currency/PPP conversion ratio

$=$ amount international dollars.

\section{Data collection and the setting}

Data collection was done by interviews conducted with (caregivers of) children with clubfeet $(n=51)$. In addition to this, healthcare professionals $(n=11)$ were also interviewed for data collection. The settings for data collection were clubfoot clinics, rehabilitation clinics, pediatric orthopaedic outpatient clinics and an orthotist workshop. The caregivers were asked to participate in the study during the plaster application, whilst the interviewer was observing or assisting with the plaster application or when the patients came for assessment or follow up for the treatment of clubfoot. The caregivers and children were interviewed sometimes with nursing staff, but without the practitioner present, with the exception of Buenos Aires, where the practitioner helped translate Spanish to English. Effort was made to create an atmosphere during the interview where caregivers could be honest about their answers without the feeling of being judged for them. Therefore the authors tried to provide an environment where caregivers could talk privately and without distractions.

SvW speaks Spanish on a basic level and was helped by a native Spanish speaking orthopaedic surgeon who was also fluent in English. Another author (AO) speaks fluent Indonesian and conducted the interviews in Indonesia; a local interpreter was used when the caregivers only spoke the local language.

The interviews with the caregivers had a duration between 15 and 30 minutes.

In the Netherlands, Argentina and South Africa care was provided by local paediatric orthopaedic surgeons all trained in and experienced with the Ponseti treatment. In Indonesia only few orthopaedic surgeons are able to give this treatment, travel distances are far, and the treatment is expensive. Treatment in the Indonesian hospitals was provided by four experienced orthopaedic surgeons from abroad (three from the Netherlands, one from Belgium) (Figs. 3 and 4). As the surgeons from abroad are only available for four weeks each time, the Ponseti method is not feasible and the local doctors are not capable of giving this treatment yet.

\section{Statistical analyses}

All continuous data were checked for normal distribution, and for the continuous outcome measures an independent t-test was used to compare two groups and a one way analysis of variance (ANOVA) was used for analysis of more than two groups. When a statistically significant difference was found with the ANOVA a post-hoc LSD-analysis was performed to

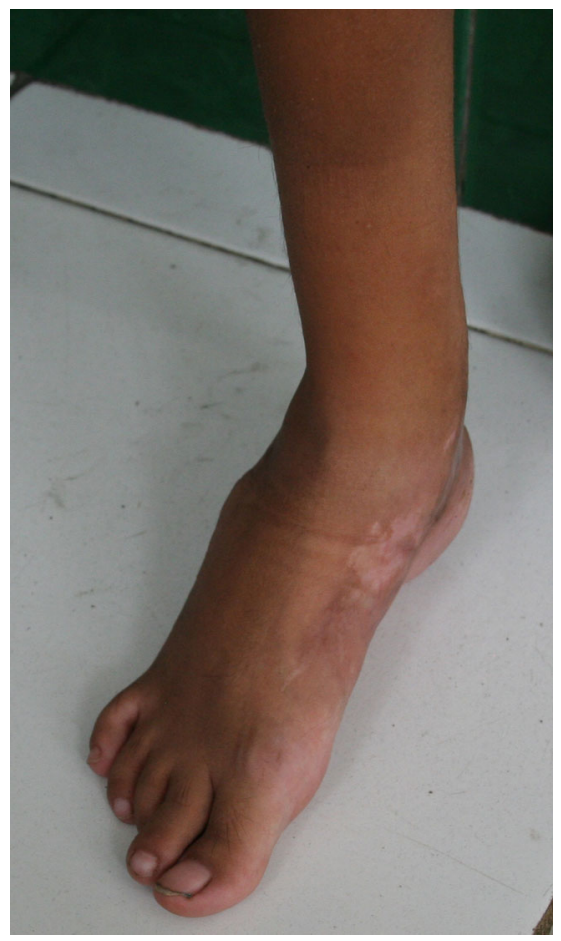

Fig. 3 Same boy as Fig. 2 after posteromedial release. The boy is able to walk and run, but the foot is very stiff due to the extensive release and scar tissue 


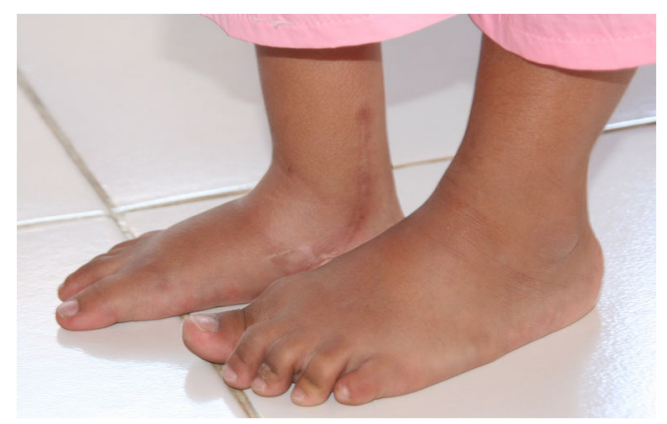

Fig. 4 Postoperative situation after a unilateral posteromedial release of a neglected clubfoot of a six-year-old girl with slight overcorrection and also a large scar and a stiff foot

see between which groups the differences existed. Pearson correlations $(\rho)$ were calculated for continuous outcome measures with a continuous predictor, and for categorical variables the Spearman correlation $\left(\rho_{\mathrm{s}}\right)$ was used. Contingency tables were made for nominal predictors to compare the differences between countries or treatment places.

As treatment should start before the walking age, which is at minimum eight months [8] we built a binary logistic regression model with a cut-off point of eight months. When the treatment started after the age of eight months it was called "neglected clubfoot". All factors were analysed as univariate and a multivariable model was built with the forward method based on likelihood ratios.

\section{Results}

\section{Treatment protocol}

The treatment of choice was the Ponseti method; only in Indonesia the majority of the patients underwent surgery for the correction of the (neglected) clubfoot. The casting period as part of the Ponseti method was comparable in duration in every country, which was six to ten weeks. The bracing protocol ranged from very strict in Amsterdam to a high percentage of loss to follow up after completion of the casting protocol in Cape Town, according to a local orthopaedic surgeon. In Indonesia the preparation for surgery and the following rehabilitation was at least 12 weeks up to a year. This was also the most expensive treatment. In Amsterdam the boots for the bracing protocols were always new, whereas in the other treatment centres the boots were re-used about four times. In Buenos Aires, the Achilles tenotomy was performed under general anaesthesia in contrast to Amsterdam and Cape Town where local anaesthesia was used.

\section{Group characteristics}

The treatment in the four countries started at different ages, as summarized in Table 1. It started at about a week after birth in
Table 1 Summary of group characteristics

\begin{tabular}{lllll}
\hline Location & $\begin{array}{l}\text { Mean age at start } \\
\text { of treatment in } \\
\text { months (standard } \\
\text { deviation) }\end{array}$ & $\begin{array}{l}\text { Mean travel } \\
\text { time for } \\
\text { hospital visit } \\
\text { in hours }\end{array}$ & $\begin{array}{l}\text { Percentage } \\
\text { of } \\
\text { monthly } \\
\text { income } \\
(\%)\end{array}$ & $\begin{array}{l}\text { Percentage } \\
\text { of boys } \\
(\%)\end{array}$ \\
\hline Amsterdam & $0.24(0.21)$ & 1.1 & 0.3 & 70 \\
$\begin{array}{l}\text { Buenos } \\
\quad \text { Aires }\end{array}$ & $0.97(0.93)$ & 3.5 & 4.0 & 50 \\
$\begin{array}{l}\text { Cape town } \\
\text { Bali }\end{array}$ & $6.83(16.77)$ & 1.9 & 1.5 & 44 \\
Sumatra & $23.63(30.55)$ & 3.5 & 11.9 & 77 \\
\hline
\end{tabular}

Amsterdam with the spread of a few days. In Buenos Aires the situation was comparable, but they generally started a little later, usually a month after birth with also a small spread. In Cape Town the differences were larger, some babies were treated just as fast as in Amsterdam, others were treated years after birth. On Bali the mean and spread of the age of start of treatment was higher. The largest spread was found on Sumatra where the mean age at the start of treatment was ten years with a spread of more than five years.

\section{Barriers to the start of treatment}

In Table 2 correlations are shown between predictors and the age when the treatment of the clubfoot started. The most important finding is the positive correlation between start of the treatment for clubfoot and the travel time to the hospital $(\rho=$

Table 2 Correlations between predictors and the age on which the treatment started

\begin{tabular}{lll}
\hline Predictors & $\begin{array}{l}\text { Correlation } \\
\text { with age at the } \\
\text { start of treatment }\end{array}$ & $\begin{array}{c}\text { Significance } \\
\text { two-tailed } \\
\text { (P-value) }\end{array}$ \\
\hline $\begin{array}{lll}\text { Total travel duration } \\
\text { a }\end{array}$ & 0.741 & 0.000 \\
$\begin{array}{l}\text { Percentage of family income spent } \\
\text { on treatment }\end{array}$ & 0.643 & 0.001 \\
$\begin{array}{l}\text { No information about treatment options } \\
\text { Supernatural beliefs }\end{array}$ & 0.434 & 0.001 \\
$\begin{array}{l}\text { Dependent on charity for the } \\
\text { arrangement of transport to the clinic }\end{array}$ & 0.393 & 0.004 \\
$\begin{array}{l}\text { No reason to postpone treatment besides } \\
\text { other medical problems }\end{array}$ & -0.373 & 0.007 \\
$\begin{array}{l}\text { Parents are informed before or at birth } \\
\quad \text { about treatment options }\end{array}$ & -0.442 & 0.000 \\
$\begin{array}{l}\text { Informed about treatment by } \\
\quad \text { ultrasound maker }\end{array}$ & -0.380 & 0.001 \\
$\begin{array}{l}\text { Diagnosis at ultrasound } \\
\text { In possession of a private car to travel }\end{array}$ & -0.357 & 0.006 \\
$\begin{array}{l}\text { Annual income } \\
\text { a }\end{array}$ & -0.324 & 0.010 \\
\hline
\end{tabular}

${ }^{\mathrm{a}}$ Pearson correlation ${ }^{\mathrm{b}}$ Spearman correlation 
$0.74, P<0.001)$. When caregivers have to travel further to the clinic, the treatment starts later. This effect was mainly found in Indonesia and South Africa. In families who had to travel for a long time to receive treatment, patients were significantly more at risk on having a neglected clubfoot when starting treatment.

The moment when caregivers are informed about the treatment options for the congenital abnormality of their child has an association with the start of treatment too. When informed early, before or at birth, the treatment is started earlier $\left(\rho_{\mathrm{s}}=\right.$ $-0.44, P<0.01$ ). Information about treatment options received by the parents later in the life of the child is associated with a later start $\left(\rho_{\mathrm{s}}=0.50, P<0.001\right)$.

When caregivers stated that they would have no reason to postpone the treatment, other than a more urgent medical problem to be treated first, this was related to a significant earlier start of the treatment $\left(\rho_{\mathrm{s}}=-0.584, P<0.001\right)$. Reasons caregivers reported to postpone the treatment of their child varied per country. In Amsterdam, where all caregivers stated that nothing or the only reason to postpone a medical treatment is if another medical condition is more important to be treated first, all the children were treated in the first weeks of their life. In Cape Town finance was an issue for $60 \%$ of the caregivers. In Indonesia various reasons were mentioned, especially the lack of information and skill of the doctor were reasons that were not mentioned in the other countries.

The public opinion about clubfeet also influences the parents in seeking treatment for their children. In the Netherlands, parents ought to do everything to provide the best care possible for their child and the social environment only reacts positively on parents seeking medical help for their children. In Buenos Aires, it was not uncommon that parents carrying a baby with plaster casts are frowned upon, because they are accused of child abuse. One of the interviews in Indonesia revealed that the daughter always stayed at home to avoid entering the public space showing her handicap. The majority in Indonesia indicated that children with clubfeet are treated differently from other children, especially when they were not able to walk.

\section{Multivariable logistic regression analysis}

When looking at the predictors for starting the treatment when there was a neglected clubfoot, we first looked at the distribution of neglected clubfoot. When the treatment started after walking age, it was labelled as a neglected clubfoot. For walking age, the minimum was used, which is eight months [11].

A univariate binary logistic regression model was built in whereby unsurprisingly the country of residence was the most important factor. As almost all neglected clubfeet were found in Indonesia we divided the countries in Indonesia and nonIndonesia. The model that fitted the best was a prediction model with belief in supernatural cause (compared to a biological cause) and distance to the treatment centre in travel hours, which increased the odds of treating a neglected clubfoot in a patient older than walking age. When we built a model, with only the data from Indonesia and neglecting the data from the other countries, these predictors were qualitatively the same, but the odds ratios were smaller and the confidence intervals larger, as expected and shown in Table 3.

Most of the caregivers on Sumatra believed that the clubfoot was given by a supernatural power, and generally started later with the treatment than caregivers who gave a biological reason or said not to know the cause. In Indonesia there was also a relation between the family income and the perceived cause of clubfeet; the lower the income, the more likely the caregiver was to believe in supernatural causes for the clubfoot.

The treatment itself was all financed by different parties: the government in Buenos Aires and Cape Town (in Cape Town families are sent an invoice according to their income scale; in practice the majority of the families do not have to contribute to the treatment), charity and the families themselves in Indonesia, and in Amsterdam treatment costs are covered completely by monthly paid insurances, which every citizen of the Netherlands is obliged to have. In all interviewed treatment places caregivers had to invest in the treatment despite the fact that none of them had to pay directly for the treatment itself. The investments consisted of transport costs, missed working days, and support for the rest of the family. Increasing the costs of the treatment in terms of percentage of the family income is associated with a later start of the treatment $(\rho=0.64, P<0.01)$. On Sumatra, the average caregiver had to invest much more than the caregivers in Amsterdam. This difference is because the family situations were different. For example, in Amsterdam there is short travel duration, short waiting times to see the healthcare professional and arrangements caregivers could make with their employers, for example, to work different hours. In Indonesia, the travel distances were generally larger, one or two caregivers had to stay for a much longer time with the child in the hospital and they had to miss work and therefore would not have an income during treatment days.

Table 3 Model for the odds ratio of treating a neglected clubfoot versus treating on time

\begin{tabular}{lll}
\hline Variable & Odds ratio $^{\mathrm{a}}(95 \% \mathrm{CI})$ & Odds ratio $^{\mathrm{b}}(95 \% \mathrm{CI})$ \\
\hline Indonesia & $16.4(1.5-184)$ & \\
Supernatural cause & $10.9(1.2-102)$ & $3.9(0.4-36)$ \\
Travel duration & $1.5(1.0-2.3)$ & $1.4(0.9-2.1)$ \\
\hline
\end{tabular}

${ }^{\text {a }}$ Logistic regression with all four countries in the model

${ }^{\mathrm{b}}$ Logistic regression with data from Indonesia only 
The higher the income of the family, the earlier the clubfoot was diagnosed, mainly because of the possibility to diagnose clubfoot with an ultrasound after 20 weeks of pregnancy. In the Netherlands this is a standard procedure, in the other investigated countries clubfeet were diagnosed at birth or later, with the exception of two mothers in Cape Town. It was not always possible to diagnose the presence of a clubfoot when an ultrasound was made. There was an association with diagnosis at an ultrasound and an earlier start of the treatment $\left(\rho_{\mathrm{s}}=\right.$ $-0.36, P<0.01)$.

\section{Discussion}

Idiopathic clubfoot is a clearly visible, relatively easily treatable congenital abnormality. With an estimated incidence of 1:1000 newborns, every year about 100,000-200,000 children are born with a clubfoot worldwide, from which about $80 \%$ are in low- or middle-income countries $[5,6]$. The majority of these feet are not treated, leaving the child disabled for the rest of their life. The situation for the caregivers of clubfoot patients and healthcare professionals differed widely amongst the countries: the Netherlands is a small, wealthy country; South Africa is a large country with huge differences in wealth amongst the population; Argentina is large and differing as well, but with a strong network of social services; and Indonesia has low healthcare insurance coverage, just like the salaries outside the cities. In Indonesia the national healthcare did not include Ponseti clubfoot care, that is why the investigated clinics are largely dependent on charity for their funding. Fortunately the covering of health care costs is changing in Indonesia, making it easier for parents to seek help, but because of the enormous amount of inhabitants (estimated 250 million) and small amount of orthopaedic surgeons (estimated 600) there will be a lack of orthopaedic surgeons when all patients suddenly are able to get the treatment they need. Especially in this setting introducing a Ponseti treatment which can be given by other health care professionals can be very helpful. Most of the patients in Indonesia required surgery for the treatment of their neglected clubfoot. The factors that were found to influence the start of treatment the most, and therefore the chance of having to treat a neglected clubfoot with a less successful outcome, were travel duration and belief in a supernatural cause for the clubfoot.

Previous mentioned barriers are other forms of clubfoot management than the Ponseti method, for example, traditional medicine. Also, beliefs that the clubfoot is caused by evil spirits, or that the disability is a result of misdeeds of the family together with societal stigma can cause a barrier for the caregivers to seek or continue the Ponseti treatment [12]. Other known barriers are lack of physician education and resources, lack of physical access to healthcare, the family situation and the physical distance to the place of treatment [13].

Important to realize is that many factors are interrelated, for example, patients from rural areas have to travel longer to the urban clinics, have a lower income, do not get any income during the days that they have to go to the hospital, and in general they are more religious as compared to the people from urban areas. Two more important differences as compared to the (over)developed countries are the financial and social burdens on a family when their child is not able to work or to marry and start a family of their own.

The influence of the mode of transport relates to this interrelation; when the caregivers were dependent on the goodwill of others for making transport possible, it resulted in a later start of the treatment. With a small distance to the clinic the caregivers have to invest less time and money in the treatment, making the start and compliance to the treatment more feasible. In Cape Town there was a shuttle service from the clinic to the closest train station (1.5 km uphill), which helped parents to access the clinic. The travel duration also has an influence on follow up; the likelihood of decreased compliance to the bracing protocol is greater when caregivers have to travel a long time to the hospital, resulting in higher relapse rates [14]. Especially in the low- and middle income setting, relapse means an extra heavy burden on the family and the healthcare system after all the investments in the treatment.

In most industrialized countries the Ponseti treatment lays under the responsibility of orthopaedic surgeons. In low- and middle-income countries non-physician healthcare professionals trained in the Ponseti method can provide effective clubfoot care as well, which is proven to improve accessibility of the treatment, making treatment available to a wider public [15].

Caregivers may experience barriers in seeking and continuing treatment for the clubfoot of their child. In many publications poverty is mentioned as a factor that impedes overcoming barriers. Therefore the financial situation of the affected families and the costs of the treatment are important issues when discussing the feasibility and barriers of the treatment of clubfoot on an international scale.

In all the investigated clinics, the Ponseti treatment was the treatment of choice; nevertheless, in Indonesia, it was more prevalent to perform surgery for the correction of the foot. This difference is due to the age of the patient population; the majority is well older than the walking age and have neglected clubfeet, which are more difficult to correct using the Ponseti method [16].

The importance of communication and parental education was emphasized in all clinics. When caregivers understood the principles of the treatment and were prepared for what was going to happen during the treatment, it made it also easier for them to invest time and money in the treatment. This is important to prevent relapse. Especially the contact with other 
caregivers of children with clubfeet and the improvements they make is of particular value [17]. All this enhanced compliance to the treatment and made the treatment more accessible. Especially in places where internet access is uncommon, for caregivers word of mouth information is an important way of gathering information about clubfoot. Publications from other countries emphasize on this aspect of the treatment as well $[14,18]$.

Based on other publications [8, 19] it might be expected that a higher amount of neglected clubfoot was found in females. However we did not observe these phenomena in our dataset either because of the small size of the dataset or because a large proportion of females with neglected clubfoot did not receive any form of treatment and are therefore not observed in our study, which was executed in treatment centres.

By using the same semi-structured interviews in four culturally, economically and demographically different countries, it was possible to compare the situation for the start of treatment in those countries. Previous reports about barriers for the treatment of clubfoot are all limited to one geological area (Uganda, India, Latin America, China, Vietnam, etc.) and differ in set-up and results; cultural and contextual differences may play a role here. The semi-qualitative and semiquantitative nature of the research gave a clear insight into "what is going on" for the people who are primarily responsible for the clubfoot patients. Although the number of interviews for every investigated clinic is relatively small, making the analyses of multiple variables difficult, the authors feel that it gave a fair view of the daily practice in the clubfoot clinics. This feeling was enhanced by checking with local healthcare professionals if the days that the interviewer was present was a "normal" day. In this study it was not possible to identify caregivers of patients who never come to a clubfoot clinic for the treatment of their child, but the barriers mentioned by the interviewed caregivers who actually visited the clinic could apply to all the caregivers of clubfoot patients.

In Argentina it was not possible to conduct the interviews individually because a language barrier existed. This was solved by the help of one of the orthopaedists with both excellent language skills in English and Spanish. This could be a possible source of bias, because the caregivers could feel that they should give the 'right' answer to their doctor. In reality caregivers responses showed no discrepancies with the responses of caregivers in the setting with the researcher alone.

It could be argued that the annual income per capita adjusted for the purchasing power parity is a flawed estimator, because it does not take into account that income is not distributed equally across the population [9]. The wealthiest families (with exception of the Netherlands) go to private hospitals, therefore the interviewed caregivers in public or charity funded hospitals in South Africa, Argentina and Indonesia all had an income below the national average. The financial investments they have to make to travel to the hospital and to miss working days are therefore larger than we could calculate in this research.

The greater distance and travel time to the place of treatment for clubfoot, the later in life the treatment of clubfoot is started. This correlation was found in Cape Town and Bali and Sumatra and confirms similar findings in Asia [18, 20], Africa [15] and the Americas [14, 21], where physical accessibility is described as a barrier for the Ponseti treatment as well. Specialized clubfoot clinics in rural areas are previously proven as a solution for overcoming this barrier [8, 22].

When caregivers of the patients with clubfeet were asked what they thought the reason was that their child has a clubfeet, some of them, especially in Indonesia, responded that the cause was 'supernatural'. This kind of belief is associated with a larger likelihood of neglected clubfoot in the child [12].

The findings about barriers to the start of treatment for clubfeet can have practical implications for the design of optimal healthcare facilities. Addressing barriers influencing the start and non-compliance for the treatment can be beneficial for the whole population served and attribute to the success of healthcare programs [13]. Therefore travel distance to the place of treatment must be taken into consideration when improving clubfoot care worldwide, just as traditional healers and midwives could be helpful addressing the beliefs about the cause of clubfeet. When caregivers know that it is not their or their child's fault and that it is treatable, this could raise public awareness for the cure of clubfeet and diminish the number of handicapped children [8, 18].

Further research is needed into overcoming the barriers for effective clubfoot care in low- and middle income countries, preferably as a part of the implementation of national programs for clubfoot in developing countries.

There are several examples of successful and sustainable implementations of clubfoot programs in low- and middle income programs $[5,8,22]$. This could be a great solution in Indonesia, because at present there is no national program giving access to clubfoot care for the whole population in need. There is a lot to win in efficient and effective clubfoot care in this country in terms of quality, awareness and accessibility, for not only the urbanized areas, but for everyone. This could apply of course also to other countries without a national program for clubfoot. Therefore we need to reach out to patients in the rural areas by overcoming the barriers, to make quality clubfoot care accessible around the globe.

\section{Conclusion}

The reasons to start treatment for clubfoot later, even after walking age when the clubfoot has become neglected, differ between and within countries. Supernatural beliefs in the cause of clubfeet and travel duration are important factors, 
however they are interrelated with other barriers, such as poverty. The largest difference we found was between Indonesia and "non-Indonesia"; this might reflect the fact that the Ponseti method is hardly available in Indonesia. When we want to improve clubfoot care worldwide we need to know the factors that influence caregivers in seeking treatment for their child and address these barriers locally.

Acknowledgments The authors would like to thank the helpful staff of the Maitland Cottage Home hospital in Cape Town, the VUmc in Amsterdam, the Garrahan hospital in Buenos Aires, the foundations Puspadi Bali, Yayasan So and Harapan Jaya in Indonesia for their assistance in facilitating the research. Also the first author (SvW) acknowledges the Leiden University Fund (LUF) for financial support for travelling to Rio de Janeiro for presenting the results at the SICOT Congress.

\section{Conflict of interest There are no conflicts of interest.}

Open Access This article is distributed under the terms of the Creative Commons Attribution 4.0 International License (http:// creativecommons.org/licenses/by/4.0/), which permits unrestricted use, distribution, and reproduction in any medium, provided you give appropriate credit to the original author(s) and the source, provide a link to the Creative Commons license, and indicate if changes were made.

\section{References}

1. Radler C (2013) The Ponseti method for the treatment of congenital club foot: review of the current literature and treatment recommendations. Int Orthop 37:1747-1753. doi:10.1007/s00264-013-20311

2. Radler C, Mindler GT, Riedl K, Lipkowski C, Kranzl A (2013) Midterm results of the Ponseti method in the treatment of congenital clubfoot. Int Orthop 37:1827-1831. doi:10.1007/s00264-0132029-8

3. Morcuende JA, Dolan LA, Dietz FR, Ponseti IV (2004) Radical reduction in the rate of extensive corrective surgery for clubfoot using the Ponseti method. Pediatrics 113:376-380

4. Jowett CR, Morcuende JA, Ramachandran M (2011) Management of congenital talipes equinovarus using the Ponseti method: a systematic review. J Bone Joint Surg Br 93:1160-1164. doi:10.1302/ 0301-620X.93B9.26947

5. Owen RM, Penny JN, Mayo A, Morcuende J, Lavy CB (2012) A collaborative public health approach to clubfoot intervention in 10 low-income and middle-income countries: 2-year outcomes and lessons learnt. J Pediatr Orthop B 21:361-365. doi:10.1097/BPB. 0b013e3283504006

6. Gupta A, Singh S, Patel P, Patel J, Varshney MK (2008) Evaluation of the utility of the Ponseti method of correction of clubfoot deformity in a developing nation. Int Orthop 32:75-79. doi:10.1007/ s00264-006-0284-7

7. Agarwal A (2014) Ponseti method for late presentation of clubfoot. Int Orthop 38:207-209. doi:10.1007/s00264-013-2147-3
8. Pirani S, Naddumba E, Mathias R, Konde-Lule J, Penny JN, Beyeza T, Mbonye B, Amone J, Franceschi F (2009) Towards effective Ponseti clubfoot care: the Uganda Sustainable Clubfoot Care Project. Clin Orthop Relat Res 467:1154-1163. doi:10.1007/ s11999-009-0759-0

9. Cavanagh P, Attinger C, Abbas Z, Bal A, Rojas N, Xu ZR (2012) Cost of treating diabetic foot ulcers in five different countries. Diabetes Metab Res Rev 28(Suppl 1):107-111. doi:10.1002/dmrr. 2245

10. World Bank (2011-2012) PPP conversion factor, GDP (LCU per international \$). International Comparison Program database. The World Bank

11. Multicentre Growth Reference Study Group (2006) WHO motor development study: windows of achievement for six gross motor development milestones. Acta Paediatr Suppl 450:86-95

12. Staheli L, Ponseti I (2009) Clubfoot: Ponseti management. Global Help

13. McElroy T, Konde-Lule J, Neema S, Gitta S, Uganda Sustainable Clubfoot Care (2007) Understanding the barriers to clubfoot treatment adherence in Uganda: a rapid ethnographic study. Disabil Rehabil 29:845-855. doi:10.1080/09638280701240102

14. Avilucea FR, Szalay EA, Bosch PP, Sweet KR, Schwend RM (2009) Effect of cultural factors on outcome of Ponseti treatment of clubfeet in rural America. J Bone Joint Surg Am 91:530-540. doi:10.2106/jbjs.h.00580

15. Tindall AJ, Steinlechner CW, Lavy CB, Mannion S, Mkandawire N (2005) Results of manipulation of idiopathic clubfoot deformity in Malawi by orthopaedic clinical officers using the Ponseti method: a realistic alternative for the developing world? J Pediatr Orthop 25: $627-629$

16. Ostadal M, Chomiak J, Dungl P, Frydrychova M, Burian M (2013) Comparison of the short-term and long-term results of the Ponseti method in the treatment of idiopathic pes equinovarus. Int Orthop 37:1821-1825. doi:10.1007/s00264-013-2033-Z

17. Boardman A, Jayawardena A, Oprescu F, Cook T, Morcuende JA (2011) The Ponseti method in Latin America: initial impact and barriers to its diffusion and implementation. Iowa Orthop J 31 : 30-35

18. Lu N, Zhao L, Du Q, Liu Y, Oprescu FI, Morcuende JA (2010) From cutting to casting: impact and initial barriers to the Ponseti method of clubfoot treatment in China. Iowa Orthop J 30:1-6

19. Gadhok K, Belthur MV, Aroojis AJ, Cook T, Oprescu F, Ranade AS, Morcuende JA (2012) Qualitative assessment of the challenges to the treatment of idiopathic clubfoot by the Ponseti method in urban India. Iowa Orthop J 32:135-140

20. Wu V, Nguyen M, Nhi HM, do Thanh V, Oprescu F, Cook T, Morcuende JA (2012) Evaluation of the progress and challenges facing the Ponseti method program in Vietnam. Iowa Orthop J 32: 125-134

21. Palma M, Cook T, Segura J, Mayo L, Morcuende JA (2013) Barriers to the Ponseti method in Peru: a two-year follow-up. Iowa Orthop J 33:172-177

22. Ford-Powell VA, Barker S, Khan MS, Evans AM, Deitz FR (2013) The Bangladesh clubfoot project: the first 5000 feet. J Pediatr Orthop 33:e40-e44. doi:10.1097/BPO.0b013e318279c61d 